\title{
Antithetic Conjuncts in Written English
}

\author{
Ann Borkin \\ University of Michigan \\ U.S.A.
}

\section{Introduction 1}

There are a number of English words and phrases used to express contrast between two consecutive sections of expository, written text. In this article, I examine a small group of these: by contrast, in contrast, conversely, on the contrary, at the same time, on the other hand and yet. On syntactic grounds, these words and phrases have been classified as conjuncts by Greenbaum, and the logical relationship that they express is classified by Greenbaum as antithetic (Greenbaum 1969). Antithetic conjuncts are, in this framework, a subset of a larger group of contrastive conjuncts which express an adversative relationship, that is, a relationship of variance between two sections of text.

The particular relationship of variance that antithetic conjuncts express is one of opposition; in Greenbaum's words, 'These indicate that what is being said is in complete opposition to what has been said before' (1969:37). However, Greenbaum's definition must be interpreted differently for different conjuncts; the kind of opposition expressed by in contrast, for example, is strikingly different from that typically expressed by on the contrary and subtly different from that expressed by conversely. And although, in an earlier issue in this journal, Yee (1975) is correct in pointing out a similarity between on the other hand and conversely it would be misleading to teach students that these two conjuncts are interchangeable in all contexts. The purpose of this paper is to describe the conditions for the appropriateness of several of the most common antithetic conjuncts in written expository English, in the expectation that such a description will be useful to teachers of English to non-native speakers.

For the purpose of this study, any two sections of text related by a conjunct will be called $X$ and $Y, X$ referring to the section preceding the conjunct and $Y$ referring to the section following the conjunct. ${ }^{2}$ Antithetic conjuncts express direct opposition between pre-conjunct section $X$ and postconjunct section $Y$, either of which can be a sentence or a larger fragment of discourse. The six conjuncts discussed here express six different kinds of opposition, ranging from polar opposition on a semantic level (i.e. hot versus cold), to opposition between arguments leading to opposite conclusions. A list of the six kinds of opposition and the conjuncts appropriate to express them follows: 
1. OPPOSITION IN COMPARISON:in contrast, by contrast, on the other hand $(\$ 2.1)$

2. SUBSTITUTION IN LOGICAL SLOTS: conversely (\$2.2)

3. CONFLICTING FORCES: on the other hand (\$2.3)

4. CONTRADICTORY INFERENCES: at the same time (\$2.4)

5. PARADOX AND DILEMMA: yet ( $\$ 2.5)$

6. OPPOSITION IN REPLACEMENT: on the contrary (\$2.6)

\section{Six Types of Opposition in English}

\subsection{Opposition in Comparison}

The conjuncts discussed in this section are used to bring into relief significant differences between two essentially similar entities or states of affairs described in pre-conjunct $X$ and post-conjunct $Y$. These differences are viewed as opposite. Often, this means that they are at opposite ends of a conventional semantic polarization, but it can also mean that an author chooses to view them as polar opposites.

\subsubsection{In contrast and by contrast}

I consider in contrast and by contrast as functionally identical, and will use the abbreviation in/by contrast to refer to both of these conjuncts.

In/by contrast typically link sections of text which describe characteristics of two entities that one would have reason to expect to be similar, viewing these characteristics as not just different but as polar or near-polar opposites. An example is found in (1):

(1) ... normal human skin fibroblasts are capable of repair replication after UV irradiation. In contrast, fibroblasts from patients with the rare hereditary skin disease xeroderna pigmentosum exhibited essentially no repair replication.

In (1), two kinds of skin fibroblasts are contrasted, one kind (normal human skin fibroblasts) having the characteristic of repair replication and another kind (fibroblasts from patients with the disease xeroderma pigmentosum) not exhibiting this characteristic. The polar opposition in (1) is thus of a positive-negative kind, in this case presence versus absence (of repair replication).

In/by contrast may also be used when the same entity or members of the same class exhibit opposing characteristics at different times. In (2), for example, in contrast is used to contrast behaviors of the same type of entity: 
(2) .... it takes many trials to teach hungry rats to press a bar for a food reward, and the rat never learns the trick if the food is not presented immediately after the bar is pressed. In contrast a rat learns to avoid poisoned food in a single trial, in spite of the fact that it may not get sick until six hours after it has eaten.

(Scientific American. May 1977, p. 113.)

Here, there is an opposition between slow learning in one situation and rapid learning in another, as well as an opposition between two aspects of the learning situations: immediate versus delayed reinforcement. Although $I$ have summarized these oppositions with the antonyms slow versus rapid, and immediate versus delayed, the relevant oppositions are actually expressed in the text by the less explicit 'many trials' versus ' a single trial,' and 'imme. diately after the bar is pressed' versus 'six hours after it has eaten.'

In/by contrast can also be used in juxtaposing two states of affairs involving completely different entities, as in (3):

(3) During the early years of prohibition ... alcohol consumption in Canada and the U.S. fell to the lowest level for which there are data. In 1969, by contrast, when general stores in Finland were allowed for the first time to sell beer, there was an immediate increase in overall consumption.

(New Society. February 1976, p. 43.)

In (3), the advent of prohibition and a sharp decline in the consumption of alcohol in North America is contrasted with the end of prohibition and an immediate rise in alcohol consumption in Finland.

The opposition central to examples (1) through (3) is understandable through conventional antonymous pairs such as presence versus absence, filled versus empty, decline versus increase, etc. However, the opposition necessary for in/by contrast is not limited to opposition expressable through a clearly defined set of semantic oppositions. This can be seen in (4), for example:

(4) Mr. Thorpe had the chance to stand up for a principle, and he chose not to take it. Suppose, by contrast, that he had had the courage to meet the forces of prurience head on and concede from the beginning his warm association with Mr. Norman Scott, and asked the whole array of British citizens to examine in their conscience as to whether there were not things in their own pasts that they could recall only with embarrassment and regret. (New Statesman. 14 May 1976, p.637.)

In (4), two courses of action are presented as representing an either-or choice of standing up for a principle or not standing up for it, that is, as opposites rather than as only two out of several courses of action. Polar opposition in this example lies not in a highly conventionalized semantic contrast but rather in the way an author views the situation he is presenting. 


\subsubsection{On the other hand}

On the other hand is often used to contrast two entities. In this function it is typically placed after the subject of the sentence, which refers to the second contrasted entity. This is true in (5), for example:

(5) A legume has a high requirement for phosphorus and potassium, although it will usually provide its own nitrogen. A grass, on the other hand, has both a large requirement for nitrogen and a smaller but still significant requirement for phosphorus and potassium.

On the other hand suggests a less sharp opposition than in/by contrast, and can also be used to express other kinds of opposition (cf. $\$ \$ 3$ and 1).

\subsection{On the contrary}

Usually, on the contrary is used as a replacive conjunct, as described in \$2.6. Much less commonly, it is used to express contrast, as in (6):

(6) Test question 13 is designed to elicit an affirmative response. On the contrary, the appropriate responses to questions $14-20$ are negative.

In this less typical use, on the contrary is similar to in/by contrast, although it expresses even more direct opposition than in/by contrast.

\subsection{Substitution in Logical Slots}

In the simplest use of conversely, the logical roles of two referents in pre-conjunct $X$ are switched in a parallel proposition contained in postconjunct $Y$. Such an example occurs in (7):

(7) In a steam engine, energy in the form of heat is converted into motion; conversely, when a meteorite enters the earth's atmosphere, motion is converted into heat. (Ewer and Latorre. A Course in Basic Scientific English, p. 190. London: Longman.)

The contrastive schema in (7) is very neat. In the two sentences, the logical roles of heat and motion with relation to the verb convert are exactly reversed; the first sentence states that heat is converted into motion in one circumstance, and the sentence with conversely states that, in another circumstance, motion is converted into heat. This role-switching is marked by conversely. ${ }^{3}$

(8) shows that role-switching of exactly the type illustrated in (7) is not essential:

(8) Medical and nursing personnel have long speculated that physicians and nurses who specialize in surgery have personality and professional pattems that can best be characterized by descrip- 
tions such as cold, efficient, verbally inhibited, domineering, and authoritarian. Conversely, their stereotype of the psychiatrist or psychiatric nurse is characterized by terms such as warm, unhumied, verbally fluent, compassionate, and uncompetitive. ( $\mathrm{T}$. D. Matarazzo and N. Wiens. The Interview: Research on Its Anatomy and Structure, p.74. Chicago: Aldine-Atherton.)

In (8), the logical role of medical and nursing personnel remains the same in $X$ and $Y$, although this logical role is represented by different grammatical and lexical elements (medical and nursing personnel in $\mathrm{X}$ and their in $\mathrm{Y}$ ). The logical role in $\mathrm{X}$ of physicians and nurses who specialize in surgery is taken over in $Y$ by the psychiatrist or psychiatric nurse; and the physicians and nurses who specialize in surgery, present in $\mathrm{X}$, is not present in $\mathrm{Y}$ at all. Rather than switching, then, there has been a substitution of referent - the substitution of the psychiatrist or psychiatric nurse in $Y$ for physicians and nurses who specialize in surgery in $\mathrm{X}$. In addition, there is a semantic opposition in the series cold $(\mathrm{X})$ versus warm $(\mathrm{Y})$, verbally inhibited $(\mathrm{X})$ versus verbally fluent $(\mathrm{Y})$, and so on.

What I have described as a "substitution of referent" is the substitution, in a logical slot in $Y$, of one referent for another that occupies the same logical slot in X. Role switching like that in the simpler example (7) can be viewed as substitution in two logical slots in $Y$. We can describe the conditions for the appropriateness of conversely relatively simply if we consider semantic opposition between antonymous predicates in $\mathrm{X}$ and $\mathrm{Y}$ (for example cold versus warm in (8) above) to also be a kind of substitution in a logical slot in $Y$, in this case substitution of one predicate for another that occupies an analogous logical slot in X. We can then stipulate that conversely is appropriate (a) only when there are more than two logical slots available in each of $X$ and $Y$, and (b) when there is substitution in at least two of these slots in Y. (In addition, a substitution of predicates must be of predicates opposable on a scale.) According to these rules, conversely in (9) is minimally appropriate, with three logical slots in $X$ and $Y$ :

(9) A relative described the first son as retarded to a distressing degree. Conversely, he described the second son as being exceptionally intelligent.

In (9), the first logical slot is filled by a relative in $X$ and he in $Y$; the second slot is filled by the first son in $X$ and the second son in $Y$; and the third is filled by retarded to a distressing degree in $\mathrm{X}$ and exceptionally intelligent in $Y$. There has been substitution in the last two slots. The rules predict that in (10), below, conversely is not appropriate, since although there is substitution in two slots, there are only two slots available.

(10) The first son is retarded to a distressing degree. ?Conversely, the second son is exceptionally intelligent.

Typically, conversely is accompanied by both role switching and semantic opposition, and the two are often accompanied by simple substitution of 
a referent as well. Grammatical parallelism is also common. The following example, then, shows conversely in a most favorable $\mathrm{X}-\mathrm{Y}$ context:

(11) In the chick oviduct estrogen stimulates the production of progesterone receptors, and tissues treated with estrogen are therefore more responsive to progesterone. Conversely, in mammals progesterone depresses the level of estrogen receptors and makes the target tissue less responsive to estrogen. (Scientific American. February 1976, p. 30.)

(11) has grammatical parallelism (Prepositional Phrase + Subject + Verb + Direct Object), role-switching of estrogen and progesterone, two cases of semantic opposition (stimulates versus depresses, more responsive versus less responsive), and a simple substitution (mammal for chick).

Finally, it should be noted that the choice of conversely is not a necessary one even when the conditions sketched above are met. In (12), which is modified from (3), the substitution of Finland for Canada and the U.S. in the same logical role, and the concurrent semantic opposition of decrease versus increase, make conversely appropriate; but in the actual text, by contrast was used instead.

(12) Overall consumption is clearly related to avallability. During the early years of prohibition, alcohol consumption in Canada and the U.S. fell to the lowest level for which there are data. Conversely, in 1969, when general stores in Finland were allowed for the first time to sell beer, there was an immediate increase in overall consumption.

In these examples, conversely emphasizes the substitutions in $\mathrm{Y}$, whereas by contrast marks a more simple and explicit notion of contrast.

\subsection{Conflicting Forces}

By conflicting forces, I mean the conflict in result or anticipated result of what is described in two consecutive sections of text. These conflicting results can be in the world outside the text, as in (13):

(13) Syria, .... is being pulled in two opposite directions by its fellowArabs. On the one hand, the conservative Arab regimes .... are still trying to bring off a rapprochement between Syria and Egypt ...

On the other hand, Libya and Algeria are trying to bring about a reconciliation between Syria and its old antagonist, Iraq. (The Economist. 29 May 1976, p. 57.)

or they can be understood only within the context of a chain of reasoning or line of argument being developed within the text itself, 4 as in (14):

(14) For measuring "high frequency" range-change behavior over a short time interval, say several hours, the continuous phase- 
tracking provided by the Doppler system tends to be more accurate. On the other hand, successive range observations made once a day over a period of many days or weeks provide a more accurate measure of "low frequency" range-change behavior.

In both cases, $X$ and $Y$ are viewed as working against each other, but in (14) the conflict is on a rhetorical level, with $X$ representing an argument in favor of one system of measurement and $Y$ representing an argument in favor of another.

The use of on the other hand in (13) differs from the use of on the other hand briefly discussed in 2.1 in that, in (13), two situations are not presented as merely being different, but as conflicting: the influence described in $\mathrm{X}$ would result in Syria being part of a conservative mid-east alliance, while the influence described in $Y$ would result in it being part of a more radical political bloc.

If the Doppler system and the ranging system were merely being compared in (14), this use of on the other hand would be like that discussed in 2.1 In this example, however, the comparison in $X$ and $Y$ contributes to an evaluation: $X$ argues for the superiority of the Doppler system, while $Y$ argues for the superionity of the ranging system. The two conclusions follow. ing from the two arguments are obviously contradictory, and it is the contradiction of these conclusions, not a semantic opposition of characteristics described in $\mathrm{X}$ and $\mathrm{Y}$, that makes this use of on the other hand different from that described in 3.1

On the other hand may suggest, but not explicitly express, conflict. In (13), for instance, direct conflict is made explicit not by the correlatives on the one hand and on the other, but by the phrase two opposite directions. The correlatives in this example merely serve to reinforce direct opposition stated elsewhere.

Because on the other hand does not unambiguously express direct opposition in every context (this is particularly the case when it is used to relate two sections of text with potentially conflicting effects in the world outside the text), the suggestion of conflict can be explicitly denied or attenuated, as it is in (15):

(15) As a result medical institutions and individual investigators operate today with two powerful sets of values and goals. On the one hand there is the pursuit and advancement of scientific knowledge. On the other [hand] there is the provision of humane and effective therapy for patients. Through a broad range of complex interactions these two sets of values and goals are harmonious, even complementary and mutually reinforcing. (Scientific American. February 1976, p. 30.)

The attenuation in (15) is only temporary, however; (15) continues with "Occasionally, however, scientific research and humane therapy can be in conflict." 


\subsection{Contradictory Inferences}

At the same time is appropriate when $X$ and $Y$ are viewed as contradictory, or, more often, when a possible inference based on $X$ and a possible inference based on $\mathrm{Y}$ are viewed as contradictory. An example of this use is found in (16):

(16) The Masovian is perhaps the best represented of all Final Paleolithic cultures on the European plain; in Poland alone more than 620 sites have yielded Masovian tool assemblages. At the same time the Masovian culture is basically similar to the Ahrensburgian, and it seems inescapable that both are sub-divisions of a single larger unit. (Scientific American. February 1976, p. 93.)

Here, a possible inference on the basis of $X$ - that the Masovian culture was of a degree of importance high enough to merit a separate and superordinate classification - is countered by a likely inference on the basis of $Y$ - that it does not merit a separate and superordinate classification. The passage goes on to make explicit the correct inference, with the statement it seems inescapable that both are sub-divisions of a single larger unit.

I view the opposition involved in (17), below, as primarily one of conflicting inferences:

(17) The Kremlin's policies towards Soviet Jews are beset with obvious contradictions. On the one hand, discrimination against Jews is constantly growing. At the same time, the authorities are placing ever bigger obstacles in the way of Jews who leave the country. (Jerusalem Post. 20 May 1976.)

In this view, a likely inference of $X$ (that Russia does not want its Jewish population) contradicts a likely inference based on $Y$ (that Russia does want its Jewish population), and the opposition in (17) is not primarily one of conflicting forces (i.e. making Soviet Jews want to leave versus not permitting them to leave). It should be noted that (17) could contain on the other hand in place of at the same time; the claim made here is that with on the other hand, the focus would be on opposite effects of Soviet policy, while with at the same time, more emphasis is placed on the contradiction in Soviet policy.

At the same time has a literal, temporal meaning, and in many cases, at the same time does not mark contradiction as much as marking a cotemporal relationship that is remarkable in some way. This is true of (18), for example:

(18) Freed from those wars, we can assert our true priorities in Asia, and, in particular, develop the crucial relationship with Japan. At the same time, the peoples of Asia, freed from the hegemonial embrace of the U.S., can work things out better for themselves, while retaining confidence that the U.S. . . intends to remain a Pacific power. (Newsweek. 7 June 1976, p. 33.) 
In (18), $X$ and $Y$ do not counter each other; rather, $X$ and $Y$ in this example represent two complementary processes working together toward a common effect.

Even when at the same time is used with the effect of opposition, the literal temporal meaning of at the same time must still be appropriate. (19), for example, is strange with at the same time.

(19) The Kremlin's policies towards Soviet Jews are beset with obvious contradictions. On the one hand, discrimination against the Jews has been consistently growing since the end of World War II. ?At the same time, the authorities will in the next year be placing even bigger obstacles in the way of Jews who leave the country.

At the same time is not entirely appropriate in (19) because the temporal meaning of this conjunct is violated, although it is appropriate in the analogous (17).

\subsection{Paradox and Dilemma}

When it appears that two apparently true propositions are contradictory, a paradox results. The paradoxical nature of contradiction may be ignored, as it is with at the same time, or it may be emphasized, as it is with yet; yet in example (16) above, for instance, would hint that the similarity of the Masovian and Ahrensburgian cultures is surprising or problematic in some way, whereas at the same time merely suggests that it may be contradictory.

Another difference between yet and at the same time is that yet is more generally used to express a concessive relationship, implying that $Y$ or an inference based on $Y$ is surprising in view of $X$. Thus, while at the same time presents $X$ and $Y$ as mutually inconsistent (a symmetrical relationship), yet typically presents $X$ as a basis on which to react to $Y$ (an asymmetrical relationship). This is so even if likely inferences based on $X$ and $Y$ are in fact mutually contradictory.

In addition to emphasizing the paradoxical nature of an apparent contradiction, yet is also appropriate to emphasize the conflict in a dilemma, that is, when one of two possible courses of action is necessary but both are viewed as intolerable or ineffective. Yet is thus particularly appropriate to emphasize the paradoxical natures of a double bind, as in (20):

(20) It's like Catch 22: no law firm wants a man that old and that expensive unless he is a partner, and yet unless he can get into some other law firm he'll never become a partner. (New Statesman. 14 May 1976, p. 645.)

Notice that in (20), the conjunction and before yet bolsters the symmetry of the paradox described in this example, although in my judgment and is not necessary here. 
In the case of irony, $X$ and $Y$ are in an asymmetrical relationship; $X$ provides the basis for a likely expectation, and $Y$ presents a situation that not only negates that expectation, but also is in some sense opposite of what one might expect. This is the case in (21), for example:

(21) Skilled doctors and researchers have posted numerous successes in dealing with trauma and acute pathology. Yet, ironically, the "modern medical miracles" have themselves contributed to the current crisis in health care (New West. 3 January 1977, p. 15.)

$Y e t$ is appropriate in ironical contexts because of its characteristics as a concessive conjunct, presenting $Y$ as unexpected in view of $X$ and further suggesting surprise and/or puzzlement. However, it would not be accurate to state that yet suggests the oppositeness of paradox; it seems to me, for instance, that if the adverb 'ironically' were not present in (21), yet would simply mark problematic or paradoxical linkage.

\subsection{Opposition in Replacement}

According to Greenbaum, replacive conjuncts (e.g. on the contrary, instead, rather) indicate that "what is being said is a replacement for what has been said before" (Greenbaum 1969:59). On the contrary is like instead and rather in that it follows one or more negative statements that describe what is not the case; but on the contrary is the only one of these which indicates that what is being said in $Y$ is the complete opposite of what has been denied in $\mathrm{X}$. Thus, in this section I discuss only on the contrary.

With on the contrary, there are several ways in which $Y$ can be opposed to $X$. Y may be both grammatically and logically an affirmative counterpart of $X$ as in (22):

(22) One thing that cannot be said of the "cohabitation rule" is that it has had insufficient attention given to it. On the contrary, sufficient attention has most certainly been paid.

Typically, however, $Y$ does not directly restate in affirmative fashion the equivalent of the denial in $X$. Instead of merely repeating the force of $X$ by an affirmative restatement, $Y$ usually makes a much stronger statement, as in $(23)$ :

(23) One thing that cannot be said of the "cohabitation rule" is that it has had sufficient attention given to it. On the contrary, official reviews of it are becoming almost annual events. (New Society. 4 March 1976, p. 474.)

Whereas in (22) $Y$ makes no claim stronger than the claim in $X$ (that sufficient attention has been paid), in (23), $Y$ suggests that the attention has been much more than sufficient. The opposition involved, then, is stronger than a simple positive-negative opposition; $Y$ pushes the opposition to a point farther along a polar scale. Similarly, in (24), a restatement opposing the notion "special circumstances" with routinely is followed by the added 
on a massive scale, which moves the opposition even further away on a scale of frequency.

(24) The work is not restricted to special circumstances. On the contrary, it occurs routinely, and on a massive scale. (J.C. McKinney and E.A. Tirgakian. Theoretical Sociology, p. 353.)

A third possibility is that the message in $Y$ can present an alternative which is logically inconsistent with the denied possibility in $\mathrm{X}$ although it is not 'opposite' in the two senses described above. An example is found in (25):

(25) One clause is not embedded within the other; on the contrary, the component clauses combine to form a new structure. (Source unknown)

The last two kinds of opposition (a statement in $Y$ moving farther on an oppositional scale than $Y$, and a statement in $Y$ logically inconsistent with what is denied in $X$ ) assume that the reader shares assumptions and knowledge with the writer in a way that the first kind (a grammatically affirmative counterpart in $Y$ of a negative statement in X) does not. (25), for example, is understandable only to those familiar with the grammatical concepts "embedding" and "conjoining," and (23) is based on the assumption that nearly annual official reviews of the cohabitation rule are more than sufficient. Similarly, in (26), the denied assertion ${ }^{5}$ and the opinion in $Y$ are not obviously contradictory; it is the use of on the contrary that forces us to interpret them as such:

(26) Professor Bayley .... convinces readers of the fundamental point ... by himself behaving with thorough conviction. This is not at all the same as saying that he writes subliterature in some way, or tries to insert himself between you and the authors he writes about. On the contrary, one of his most characteristic manoeuvres is precisely to separate out the criticism from the literature, and to rescue the work he deals with from the constructs which, he would say, have tidied and obscured them. (New Statesman. 14 May 1976, p. 652.)

\section{Concluding Remarks}

I have adopted Greenbaum's definition of the logical function of antithetic conjuncts, which I repeat here: "These [antithetic conjuncts] indicate that what is being said is in complete opposition to what has been said before." As we have seen, this definition is to be interpreted differently for different conjuncts. With on the contrary, what is being said is not in complete opposition to what has been said before, but rather to what has been denied before. In/by contrast and conversely express contrast without contradiction or conflict; but at the same time is appropriate to suggest contradiction, and with yet $\mathrm{X}$ and $\mathrm{Y}$ are viewed as contradictory to the extent of paradox. With on the other hand (when not used as a simple comparative), the relevant opposition is found in two kinds of effects of $X$ and 
$Y$ - either in the empirical effects of the states of affairs described in $X$ and $Y$ or in the rhetorical effects of the states of affairs described in $X$ and $Y$.

Thus, it seems that antithetic conjuncts do not represent a functionally similar class on the same level as concessives (cf. Quirk 1954, Greenbaum 1969) or replacives (Greenbaum 1969). Rather, the nature of the direct opposition implied by each antithetic conjunct is understood with regard to its function within a text ${ }^{6}$ : to compare (in/by contrast, conversely, and on the other hand), to indicate a noteworthy correlation (at the same time), to indicate a countered expectation (yet), to replace a negative statement with a positive one (on the contrary), and to indicate different empirical or rhetorical effects (on the other hand). Other conjuncts not discussed here share these same general functions; what distinguishes the conjuncts examined in this paper is their focus on the semantic aspect of direct opposition within one of these more general textual functions.

\section{Notes}

1. I would like to thank Larry Selinker for valuable editorial help, and for helping me clarify the distinction between contradictory inferences, and paradox and dilemma.

2. "The section following the connective" should be understood as "the sentence with the connective plus any additional sentences that represent the second part of the contrast." This understanding is necessary because not all the connectives under discussion here always occur in sentenceinitial position.

3. It should be noted that conversely is not appropriate in a narrative sequence, with a notion of reciprocal actions comprising what is viewed as a single event, as in (i):

(i) The rebels initiated an attack on the Nationalists on Sunday morning. *Conversely, the Nationalists immediately mounted a counterattack against the insurgents.

Example (i) can be contrasted with (ii), in which the events described in $X$ and $Y$ are viewed as separate in time and not causally related:

(ii) The South initiated an attack on the North in 1812. Conversely, in the second civil war fifty years later, the North was the first to attack the South.

Here, conversely is appropriate.

4. This distinction is similar to a distinction called external versus internal by Halliday and Hasan (1976), but it is not equivalent; thus Halliday and Hasan would consider all uses of on the other hand in this section to be external. 
5. Notice that the grammatically negated assertion in this example is 'this is the same as ....', while the pragmatically denied assertion is the writes subliterature in some way, or tries ...? Thus, on the contrary here does not oppose $Y$ to 'this is the same as saying ...', but rather to the pragmatically denied assertion.

6. This is, in fact, how they are treated in Halliday and Hasan (1976).

\section{References}

Arapoff, N. 1968. The semantic role of sentence connectors in extra-sen tence logical relationships. TESOL Quarterly 4. 243-253.

Ballard, D.L., Conrad, R.J. and Longacre, R.E. 1974. The deep and surface grammar of interclausal relations. Advances in tagmemics, ed. by R.M. Brend, 307-355. Amsterdam: North-Holland Publishing Co.

Borkin, A. 1979. Expectancy-reversal connectives in written English. Forthcoming.

Curme, G.O. 1931. Syntax. Boston: D.C. Heath.

Greenbaum, S. 1969. Studies in English adverbial usage. Coral Gables: University of Miami Press.

Halliday, M.A.K., and Hasan, R. 1976. Cohesion in English. London: Long. man.

Lakoff, R. 1971. If's, and's and but's about conjunction. Studies in linguistic semantics, ed. by C. Fillmore and D' T. Langendoen, 115-149. New York: Holt, Rinehart, and Winston.

Longacre, R.E. 1970. Sentence structure as a statement calculus. Language 46. $783-815$.

Quirk, R. 1954. The concessive relation in Old English poetry. New Haven: Yale University Press.

Winter, E.O. 1971. Connection in science material. Science and technology in a second language, 45-52. London: Centre for Information on Language Teaching and Research.

Yee, C.T.S. 1975. Sequence signals in technical English. RELC Journal 6:2. 63-101.

Young, R.E., Becker, A.L. and Pike, K.L. 1970. Rhetoric: discovery and change. New York. Harcourt, Brace \& World. 